American J. of Engineering and Applied Sciences 3 (2): 464-469, 2010

ISSN 1941-7020

(C) 2010 Science Publications

\title{
Effect of Two Interacting Localized Defects on the Critical Load for Thin Cylindrical Shells Under Axial Compression
}

\author{
${ }^{1}$ Abdellatif Khamlichi, ${ }^{1}$ Jalal El Bahaoui, ${ }^{1}$ Larbi El Bakkali, ${ }^{1}$ Mohammed Bezzazi and ${ }^{2}$ Ali Limam \\ ${ }^{1}$ Modeling and Simulation of Mechanical Systems Laboratory, Department of Physics, \\ Faculty of Sciences at Tetouan, University Abdelmalek Essaadi, \\ BP. 2121, M'hannech, 93002 Tetouan, Morocco \\ ${ }^{2}$ Civil and Environmental Engineering Laboratory, Institute of Applied Sciences at Lyon, \\ 20 Coulomb Edifice, Albert Einstein Avenue, 69621Villeurbanne Cedex, France
}

\begin{abstract}
Problem statement: Many modern structures are made from thin shells. Design of these elements depends to a large extent on their buckling behavior which is hugely affected by the initial geometric imperfections. Approach: For axially compressed isotropic circular cylindrical shells, axisymmetric localized geometric imperfections were found to reduce severely the buckling strength. Among various axisymmetric shapes of localized defects that were investigated, the entering triangular form was recognized to yield the most adverse case. Since multiple localized defects may be present in the same shell structure and interact, studying their mutual effect on the buckling load is of great importance for shell design. Results: In this study, the effect of two interacting entering triangular localized axisymmetric initial geometric imperfections on shell buckling strength under uniform axial compression was modeled by means of the finite element method. A special software package which was dedicated to buckling analysis of quasi axisymmetric shells was used in order to compute the buckling load either via the linear Euler buckling analysis or through the full non linear iterative procedure. A set of five factors including shell aspect ratios, defect characteristics and the distance separating the localized initial geometric imperfections had been found to govern the buckling problem. A statistical approach based on the Taguchi method was used then to study their relative influence on the buckling load reduction. It was shown by comparison with the single imperfection case that further diminution of the critical load was obtained. Conclusion/Recommendations: In the range of investigated parameters, the distance separating the localized geometric imperfections and imperfection wavelength were found to yield major influences on the critical load. Further studies must be performed in order to assess shell buckling strength in the presence of more than two defects and to state the relative influence of the intervening factors.
\end{abstract}

Key words: Buckling, finite element method, shells, localized geometric imperfections, Taguchi method

\section{INTRODUCTION}

Thin shells are used in many fields such as structural elements (silos, tank). Whatever the manufacturing process used for this type of structures, the final geometry is never perfect. Defects affecting the shell initial form known as geometric imperfections disturb the ideal desired shell geometry. Control of manufacturing processes of shells and their optimization makes it certainly possible today to decrease these imperfections, but they could never be completely eliminated since, even if at first guess the geometry seems to be perfect, precise measurements enable always to detect defects having in general a magnitude of the same scale order than shell thickness.

During service life, shell structures may be subjected to various kind of loading, such as axial compression, external/internal pressure, flexure or torsion. For thin cylindrical shells under uniform axial compression, the buckling strength constitutes always the most adverse design issue. Calculation of the buckling load as it could be affected by the presence of various kinds of initial geometric imperfections represents hence a crucial task. The pursued objective is

Corresponding Author: Abdellatif Khamlichi, Modeling and Simulation of Mechanical Systems Laboratory,

Department of Physics, Faculty of Sciences at Tetouan, University Abdelmalek Essaadi, BP. 2121, M'hannech, 93002 Tetouan, Morocco 
to know how to perform shell structural design with pertinent and relevant margins of safety.

Several studies have been reported in the literature which deals with the effect of imperfections on strength buckling of thin shell structures. Arbocz and Babcock (1969) have studied experimentally buckling of cylindrical shells subjected to general imperfections. They have shown that a huge reduction of the buckling critical load could be obtained. Koiter (1982) has given a review study about the effect of geometric imperfections on shell buckling strength. Other extensive investigations have been achieved in the particular field of shell buckling as it could be influenced by initial geometric imperfections: Koiter (1982); Yamaki (1984); Arbocz (1987); Bushnell (1989); Godoy (1993) and Combescure and Galletly (1999). They have dealt with the effect of both distributed and/or localized imperfections on reduction of the buckling load.

All the previous literature agrees on the fact that imperfections reduce drastically the buckling load of elastic cylindrical shells when subjected to axial compression. The obtained reduction depends however on the nature of initial geometric imperfections that are present in the shell structure. It has been found also that reduction of the buckling load is, in general, more severe in case of distributed imperfections than for localized ones.

Imperfections for which maximum reduction of the buckling load is obtained could be artificial and purely theoretical like for instance the well known generalized Koiter imperfection: Koiter (1982). They might hence never be encountered in practice in case of real shells. Therefore, modern investigation in the filed of shell buckling has been motivated largely by the analysis of buckling strength in the presence of typical imperfections obtained from modal analysis of measured data or by considering realistic imperfection shapes such as those resulting from welding operations performed during assembling of shell parts.

Steel silos and tanks are constructed from plates which are rolled to obtain the correct curvature and subsequently welded together to form strakes. The strakes are brought together then to assemble by welding the complete shell structure. At circumferential welds localized geometric imperfections develop. The welding profile can vary from one shell to another but a common feature of welds is that their geometry can be characterized by a small number of parameters which are associated to the amplitude and wavelength of weld defects. Measurements have revealed that mostly axisymmetric imperfections occur in shell structures assembled by welding, Ding et al. (1996).
Circumferential weld-induced imperfections were found to have a great influence on buckling of thinwalled cylindrical shell structures. Hutchinson et al. (1971); Amazigo and Budianski (1972) and Gusic et al. (2000) investigated localized axisymmetric imperfections and have shown that a single axisymmetric imperfection is sufficient to yield large effect on buckling strength of thin shells.

Combining shell theory with actual field imperfection measurements, Pircher et al. (2001) have found that three parameters governed the shape of the surveyed weld imperfections: the amplitude (depth), the wavelength (width) and the roundness. This last has been found to have small influence in comparison with the two first.

Using an analytical approach which is based on Arbocz equations, Arbocz (1987) and Khamlichi et al. (2004) have considered a parabolic localized imperfection and have obtained large reduction of the buckling load for thin axisymmetric cylindrical shell under uniform axial compression. Using finite element modeling of shell buckling Mathon and Limam (2006) has compared the relative influence of several localized imperfections on reduction of the buckling load of shells subjected to axial compression or to flexure. He has shown that a triangular imperfection shape has the most severe effect on buckling strength.

Considering the combined effect resulting from localized geometric imperfections and residual stresses Hübner et al. (2006) has recently investigated the case of large steel cylinders with patterned welds.

In almost all the previous study only single geometric imperfections were considered. The objective of this study is to investigate how two interacting localized imperfections would affect the shell buckling strength. The localized geometric imperfections considered are assumed to have an entering triangular form. It was found by Mathon and Limam (2006) that the entering configuration (peak of the geometric imperfection is towards the shell axis of symmetry) yields the most adverse case as to buckling strength reduction in comparison with the outgoing configuration (peak of the geometric imperfection is outwards the shell axis of symmetry). The triangular geometric imperfection has a shape which can be characterized by only tow parameters: The amplitude and the width denoted also wavelength.

Investigation of the relative effect of the intervening factors on the shell buckling load is performed following two stages. At first, the triangular geometric imperfection is considered alone and then in the situation where two defects having this form are interacting. In this second situation, the distance separating the two defects is an additional parameter. To 
these three parameters one should add the shell aspect parameters: Radius over thickness and length over thickness. Thin axisymmetric cylindrical shells made of homogeneous and isotropic elastic material are considered. They are assumed to deform under a purely axisymmetric strain state when they are subjected to axially uniform compressive loads.

The objective pursued is to quantify the relative influence of factors. This is performed in order to guess which factor would be preferably to act on in order to enhance the shell buckling resistance.

In order to limit the total number of simulations, a design of experiment method using Taguchi (1986) approach is applied. Three levels for each of the five intervening factors have been selected.

\section{MATERIALS AND METHODS}

Shell equations corresponding to Sanders model and incorporating the effect of initial imperfections, Markus (1988), are used to analyze the effect of initial geometric imperfections on shell buckling strength for the particular case of thin circular cylindrical shells subjected to quasi-static uniform compressive loads. A variant of this model has been used by Combescure and Galletly (1999) in order to perform finite element modeling of shell buckling. Relevant modeling of geometrically imperfect shell equations has then been carried out by this author who has developed Stanlax software package (called earlier INCA). Stanlax is based on an analytical expansion in terms of the circumferential wave number and finite element modeling of axial dependant quantities. The initial imperfections are included in shell model formulation under the assumption of small perturbations to the shell geometry.

This software was validated by comparison of the obtained bulking results with those produced by other commercial finite element buckling software.

For cylindrical axisymmetric shells undergoing axial compression a special element designated Coque was developed. It is used in the following in order to model the imperfect axisymmetric cylindrical shell having one or two localized geometric imperfections. Stanlax offers either a linear Euler buckling analysis mode or a full non linear iterative computation of the buckling load. For shells under axial compression, it was shown that a linear Euler calculus is sufficient.

The shell material is linear elastic having Young's modulus E and Poisson's ratio and v. The defects are localized in the median zone of the shell length and sufficiently far from the shell ends in order to avoid any interaction with the boundary conditions. The selected boundary conditions are those corresponding to clamped shell ends.

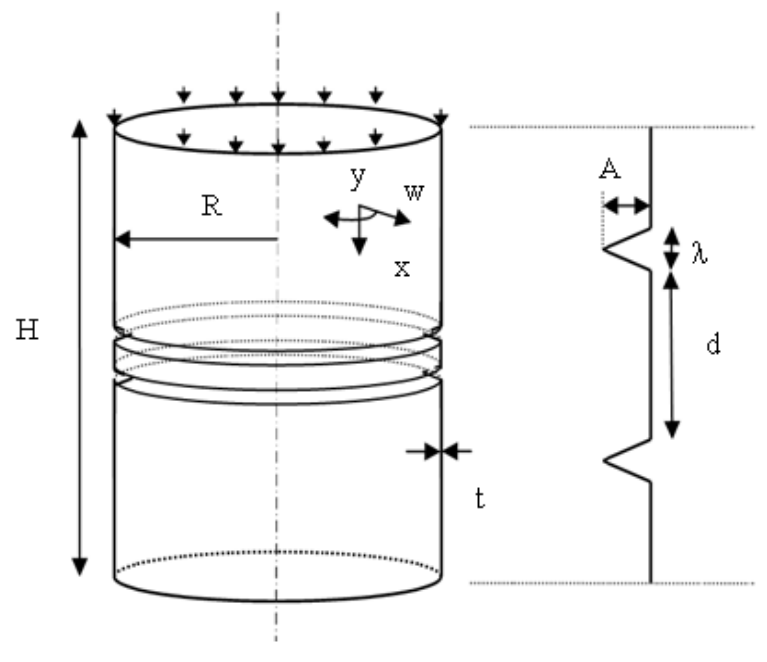

Fig. 1: Geometry of the imperfect cylindrical shell showing two localized defects

During the whole study the shell radius is maintained constant at the value $\mathrm{R}=135 \mathrm{~mm}$ while the other parameters are varied. As shown in Fig. 1, parameters t, H, A designate respectively shell thickness, shell length, defect amplitude and distance separating the two localized geometric imperfections.

To clarify the presentation, the following non dimensionalized parameters obtained form the imperfect shell factors monitoring the shell buckling strength are introduced:

- $\mathrm{R} / \mathrm{t}$ radius to thickness ratio

- $\mathrm{H} / \mathrm{R}$ length to radius ratio

- $\mathrm{A} / \mathrm{t}$ defect amplitude parameter

- $\mathrm{d} / \mathrm{R}$ defect interval scale to radius ratio

- $\mathrm{K}=\lambda /(1.72 \sqrt{\mathrm{Rt}})$ parameter fixing the defect wave length $\lambda$

\section{RESULTS}

Let's consider a single triangular geometric imperfection located at the mid height of the shell for which geometric and material properties are given by: $\mathrm{R}=135 \mathrm{~mm}, \mathrm{H}=405 \mathrm{~mm}, \mathrm{t}=0.09 \mathrm{~mm}, \mathrm{E}=7 \times 10^{10} \mathrm{~Pa}$ and $v=0.3$. In this case the classical buckling load is $\sigma_{\mathrm{cl}}=28.233 \times 10^{6} \mathrm{~Pa}$. When, the imperfection amplitude is fixed at $\mathrm{A} / \mathrm{t}=1$ and its wavelength at $\lambda=15 \mathrm{~mm}$, Fig. 2 presents the evolution of the buckling load ratio $\sigma_{\mathrm{cr}} / \sigma_{\mathrm{cl}}$ as function of the number of elements with $\sigma_{\mathrm{cr}}$ the actual critical load and $\sigma_{\mathrm{d}}$ the classical buckling 
load defined as $\sigma_{\mathrm{cl}}=\frac{\mathrm{E}}{\sqrt{3\left(1-v^{2}\right)}} \times \frac{\mathrm{t}}{\mathrm{R}}$. A total number of 100 elements were found sufficient to guarantee FEM model convergence. Another study on the influence of the number of circumferential harmonics on FEM results has enabled to conclude that 25 harmonics are enough to guarantee convergence in the example considered. Other results not shown here have demonstrated that for all single or interacting imperfection cases a total number of 300 elements and a total number of 30 harmonics guarantee well convergence of the finite element model.

The effect of a single localized geometric imperfection has been investigated at first in order to determine the most severe defect characteristics as to the buckling load reduction, El Bahaoui (2007). These characteristics have then been used to estimate the effect of two interacting defects on the shell buckling load. In the following the single defect parameters corresponding to the most adverse case are referred to as intermediate values as indicated in Table 1. Table 1 gives also the list of parameters levels that have been considered in the analysis of shell buckling under the coupling situation of localized geometric imperfections: Lower threshold, intermediate value and higher threshold.

Based on Table 1, a parametric study regarding the influence of two interacting defects has been conducted. This was performed according to a design of experiment method using five factors and three levels for each factor such as they are given by the Taguchi table $\mathrm{L}_{27}\left(3^{13}\right)$. The model so considered is hence linear without any interaction between factors. It has been shown a posteriori that there is no need to consider interaction between factors as the residuals are very small.

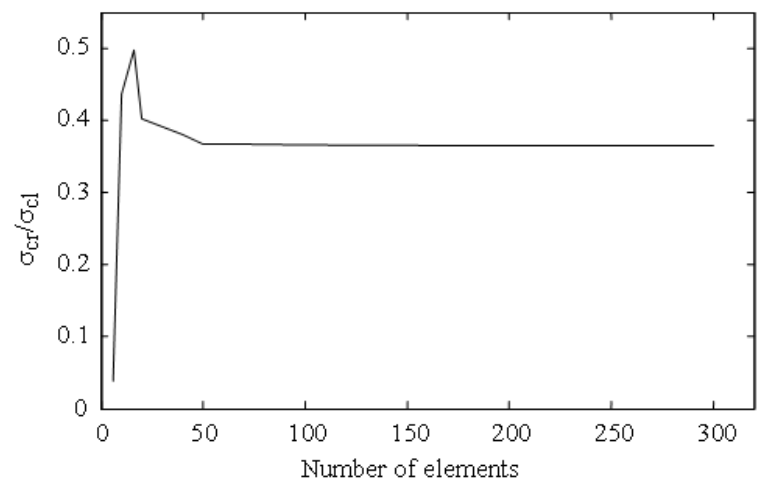

Fig. 2: FEM convergence as function of the number of elements
Stanlax software enables for each combination associated to a given line of the Taguchi table $\mathrm{L}_{27}\left(3^{13}\right)$ to compute the critical load. A total set of 27 numerical simulations have been performed. The results are given in Table 2.

Subsequently Analysis Of Variance (ANOVA) has been performed in order to determine the relative influence of each factor.

By imposing the shell aspect ratio $\mathrm{R} / \mathrm{t}$ to be greater than 450 for thin shell approximation to be valid, it has been shown in El Bahaoui (2007) that the most adverse case, with regards to the shell buckling strength when considering a single entering triangular geometric imperfection, is obtained for: $\mathrm{R} / \mathrm{t}=450$, $\mathrm{H} / \mathrm{R}=3, \mathrm{~K}=2.5$, the obtained results expressed as the actual critical load divided by the classical critical load versus the parameter $\mathrm{A} / \mathrm{t}$ are presented in Fig. 3. The continuous curve gives the results associated to a single defect. The dashed curve gives in the same conditions of parameters values the results for two interacting triangular geometric imperfections.

Table 1: Ranges of variation of the considered factors

\begin{tabular}{lllrll}
\hline Parameter & $\mathrm{K}$ & $\mathrm{A} / \mathrm{t}$ & $\mathrm{R} / \mathrm{t}$ & $\mathrm{H} / \mathrm{R}$ & $\mathrm{d} / \mathrm{R}$ \\
\hline Lower threshold & 1 & 2.0 & 450 & 1 & 0.000 \\
Intermediate value & 2 & 2.5 & 1000 & 2 & 0.370 \\
Higher threshold & 3 & 3.0 & 1500 & 3 & 0.741 \\
\hline
\end{tabular}

Table 2: Simulation data layout according to Taguchi $\mathrm{L}_{27}\left(3^{13}\right)$ orthogonal array

\begin{tabular}{|c|c|c|c|c|c|c|}
\hline $\begin{array}{l}\text { Simulation } \\
\text { number }\end{array}$ & $\mathrm{A} / \mathrm{t}$ & $\mathrm{d} / \mathrm{R}$ & K & $\mathrm{R} / \mathrm{t}$ & $\mathrm{H} / \mathrm{R}$ & $\sigma_{\mathrm{cr}} / \sigma_{\mathrm{cl}}$ \\
\hline 1 & 2.0 & 0.000 & 1 & 450 & 1 & 0.287 \\
\hline 2 & 2.0 & 0.000 & 2 & 1000 & 2 & 0.222 \\
\hline 3 & 2.0 & 0.000 & 3 & 1500 & 3 & 0.213 \\
\hline 4 & 2.0 & 0.370 & 1 & 1000 & 3 & 0.240 \\
\hline 5 & 2.0 & 0.370 & 2 & 1500 & 1 & 0.247 \\
\hline 6 & 2.0 & 0.370 & 3 & 450 & 2 & 0.188 \\
\hline 7 & 2.0 & 0.741 & 1 & 1500 & 2 & 0.229 \\
\hline 8 & 2.0 & 0.741 & 2 & 450 & 3 & 0.261 \\
\hline 9 & 2.0 & 0.741 & 3 & 1000 & 1 & 0.809 \\
\hline 10 & 2.5 & 0.000 & 1 & 1000 & 1 & 0.254 \\
\hline 11 & 2.5 & 0.000 & 2 & 1500 & 1 & 0.192 \\
\hline 12 & 2.5 & 0.000 & 3 & 450 & 3 & 0.185 \\
\hline 13 & 2.5 & 0.370 & 1 & 1500 & 3 & 0.293 \\
\hline 14 & 2.5 & 0.370 & 2 & 450 & 1 & 0.371 \\
\hline 15 & 2.5 & 0.370 & 3 & 1000 & 2 & 0.733 \\
\hline 16 & 2.5 & 0.741 & 1 & 450 & 2 & 0.355 \\
\hline 17 & 2.5 & 0.741 & 2 & 1000 & 3 & 0.184 \\
\hline 18 & 2.5 & 0.741 & 3 & 1500 & 1 & 0.686 \\
\hline 19 & 3.0 & 0.000 & 1 & 1500 & 1 & 0.223 \\
\hline 20 & 3.0 & 0.000 & 2 & 450 & 2 & 0.165 \\
\hline 21 & 3.0 & 0.000 & 3 & 1000 & 3 & 0.166 \\
\hline 22 & 3.0 & 0.370 & 1 & 450 & 3 & 0.574 \\
\hline 23 & 3.0 & 0.370 & 2 & 1000 & 1 & 0.300 \\
\hline 24 & 3.0 & 0.370 & 3 & 1500 & 2 & 0.135 \\
\hline 25 & 3.0 & 0.741 & 1 & 1000 & 2 & 0.733 \\
\hline 26 & 3.0 & 0.741 & 2 & 1500 & 3 & 0.172 \\
\hline 27 & 3.0 & 0.741 & 3 & 450 & 1 & 0.619 \\
\hline
\end{tabular}


Am. J. Engg. \& Applied Sci., 3 (2): 464-469, 2010

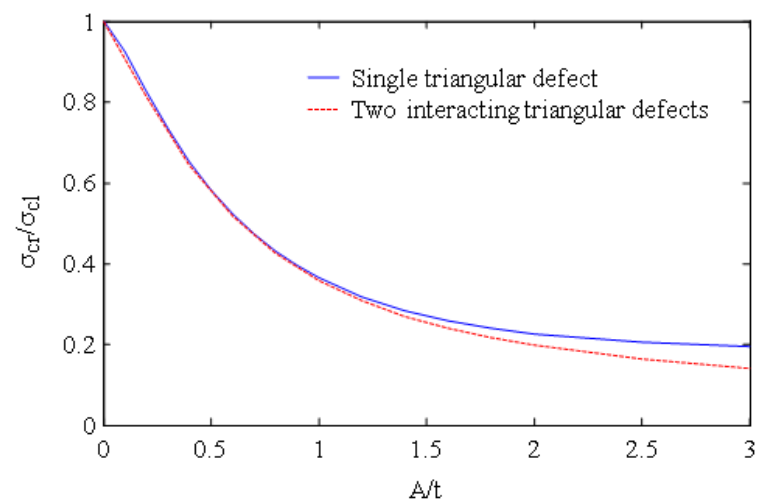

Fig. 3: Comparison between a single defect and two interacting defects

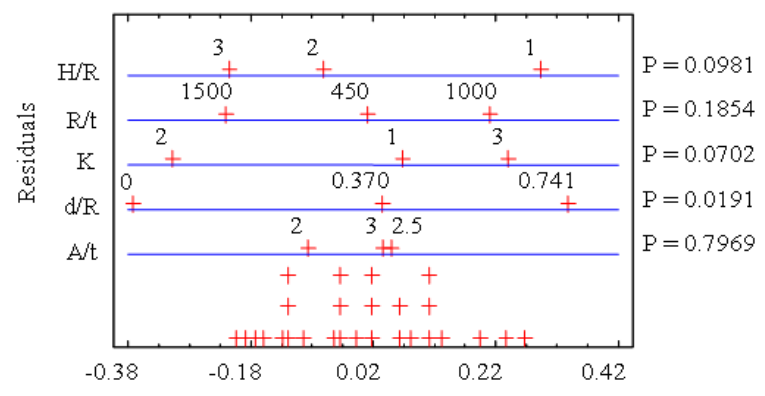

Fig. 4: Multifactor ANOVA diagram performed on the five factors

The effect of two interacting geometric imperfections is not very important when the parameter $\mathrm{A} / \mathrm{t}$ is small. But, when $\mathrm{A} / \mathrm{t}$ increases $(\mathrm{A} / \mathrm{t}>1)$ the two geometric imperfection configuration yield an obvious reduction of the shell buckling strength as compared with the single geometric imperfection effect. The most adverse reduction of the shell buckling load $\sigma_{\mathrm{cr}} / \sigma_{\mathrm{cl}}$ passes from 0.20 in the case of a single defect to only 0.16 in the case of two interacting defects.

Figure 4 presents the obtained ANOVA diagram performed on simulation results as given in Table 2 . One can notice that the relative distance between the two localized defects $\mathrm{d} / \mathrm{R}$ gives the lowest probability which is equal to $1.91 \%$. This percentage is well below the other percentages associated to the other remaining parameters of the problem: A/t (79.69\%), K (7.02\%), $\mathrm{R} / \mathrm{t}(18.54 \%)$ and $\mathrm{H} / \mathrm{R}(9.81 \%)$. This signifies that, in the range of parameters values investigated here when considering the situation of two coupled defects, the relative distance between the two geometric imperfections is the most important parameter. It is followed by the geometric imperfection wavelength and by the shell aspect ratios. The defect amplitude has the lowest influence on shell buckling loads.
Regarding the intrinsic influence of the geometric imperfection amplitude, it is well known that this parameter monitors to a large extent the shell buckling strength as it is shown for example in Fig. 3 in the range $A / t \in[0,3]$. But, in the range of parameters values considered here $A / t \in[2,3]$ this parameter has only a reduced influence as one could notice that form the plateau present in the buckling curves, Fig. 3.

According to the multifactor analysis of variance ANOVA, on can verify that the method of Taguchi which has been applied here without taking into account interaction between factors is well justified since the residuals are very small (not exceeding $8 \%$ ).

\section{DISCUSSION}

Studying the buckling load for the case of a single triangular entering geometric imperfection, as function of wavelength and amplitude parameters shows always that there are only small effects associated to parameters $H / R$ and $R / t$ in the range of thin shells. It had been shown also that the wavelength $\mathrm{K}=2.5$ yields the most adverse case, El Bahaoui (2007). Fixing the imperfection wavelength at this value, the most important parameter left in case of a single geometric initial imperfection is the imperfection amplitude. Its effect stabilizes however in the most dangerous interval of amplitudes $\mathrm{A} / \mathrm{t} \in[2,3]$ as the results show in general the existence of a plateau in the curve giving the buckling load $\sigma_{\mathrm{cr}} / \sigma_{\mathrm{cl}}$ as function of $\mathrm{A} / \mathrm{t}$.

Studying the buckling load for the case of two triangular entering geometric imperfections, as function of the above parameters plus the distance separating the localized defects has shown the same conclusions regarding the effects of shell ratio parameters, the imperfection amplitude and wavelength. This gives details why it is important to analyze effect of parameter $\mathrm{d}$ on the buckling strength within the worst interval of amplitudes $\mathrm{A} / \mathrm{t} \in[2,3]$. The pursued objective is to help from a practical point of view determining the ideal height for welding strakes which allows maximizing the bulking strength of shell assembly.

Fixing for instance the parameters: $\mathrm{R}=135 \mathrm{~mm}$, $\mathrm{A} / \mathrm{t}=2, \mathrm{~K}=2.5, \mathrm{R} / \mathrm{t}=450, \mathrm{H} / \mathrm{R}=3$ and varying the distance separating the two localized imperfections from $\mathrm{d}=50-150 \mathrm{~mm}$ increases the reduced buckling load from $0.17-0.21$. The gain is significant and can even be higher for other values of parameters.

To assess these results further parametric studies are needed. Investigating the effect of more than two defects and considering complete ranges of parameters will be very useful. 


\section{CONCLUSION}

Numerical simulations based on the finite element method have been performed in order to quantify shell buckling load reduction in the presence of localized defects. Elastic thin cylindrical shells subjected to axial compression and having one or two axisymmetric defects of entering triangular form have been analyzed. A set of five factors intervening in the problem have been considered. A parametric study according to Taguchi method of design of experiment has then been performed in order to determine their relative influence on the shell buckling strength.

It has been shown that two interacting defects could yield further reduction of the critical load in comparison with the single defect case. In the range of parameters investigated, the distance separating the two triangular geometric defects has been found to have the major influence on critical load reduction. It is followed by the defect wavelength and by the shell aspect ratios. The defect amplitude has the lowest influence on shell buckling loads.

\section{ACKNOWLEDGMENT}

The researchers would like to warmly thank Professor A. Combescure from INSA at Lyon in France for having provided freely the software package Stanlax.

\section{REFERENCES}

Amazigo, J.C. and B. Budianski, 1972. Asymptotic formulas for the buckling stresses of axially compressed cylinders with localized or random axisymmetric imperfections. J. Applied Mech., 93: 179-184.

Arbocz, J., 1987. Post-Buckling Behavior of Structures, Numerical Techniques for more Complicated Structures. Buckling and Post-Buckling of Structures, Springer-Verlage, Berlin, ISBN: 978-3540-18312-9.

Arbocz, J. and C.D. Babcock, 1969. The effect of general imperfections on the buckling of cylindrical shells. ASME J. Applied Mech., 36: 28-38.

Bushnell, D., 1989. Computerized analysis of shells. Mechanics of Elastic Stability. Kluwer Academic Publishers, Dordrecht, Boston, London, ISBN: 13: 978-9024730995.

Combescure, A. and G.D. Galletly, 1999. Plastic buckling of complete toroidal shells of elliptical cross-section subjected to internal pressure. ThinWalled Struct., 34: 135-146. DOI: 10.1016/S02638231(99)00006-3
Ding, X., R. Coleman and J.M. Rotter, 1996. Technique for precise measurement of large-scale silos and tanks. J. Survey. Eng., 122: 15-25.

El Bahaoui, J., 2007. Buckling of thin cylindrical shells: analysis of the effect of a localized imperfection. In French, Master Memory, FS Tetouan, Morocco.

Godoy, L.A., 1993. On loads equivalent to geometrical imperfections in shells. J. Eng. Mech. ASCE., 119: 186-190.

Gusic, G., A. Combescure and J. F. Jullien, 2000. The influence of circumferential thickness variations on the buckling of cylindrical shells under external pressure. Comput. Struct., 74: 461-477. DOI: 10.1016/S0045-7949(99)00053-X

Hübner, A., J.G. Teng and H. Saal, 2006. Buckling behavior of large steel cylinders with patterned welds. Int. J. Pressure Vessels Pip., 83: 13-26. DOI: 10.1016/j.ijpvp.2005.10.003

Hutchinson, J.W., R.C. Tennyson and D.B. Muggreridge, 1971. Effect of a local axisymmetric imperfection on the buckling of a cylindrical shell under axial compression. AIAA J., 9: 48-52.

Khamlichi, A., A. Limam and M. Bezzazi, 2004. Buckling of elastic cylindrical shells considering the effect of localized axisymmetric imperfections. Thin-Walled Struct., 42: 1035-1047. DOI: 10.1016/j.tws.2004.03.008

Koiter, W.T., 1982. The application of the initial postbuckling analysis to shells. Proceedings of the State-of-The-Art Colloquium on Buckling of Shells, May 6-7, Springer Verlage, Stuttgart, West Germany, Germany, pp: 3-17.

Markus, S., 1988. The mechanics of vibrations of Cylindrical Shells Studies in Applied Mechanics, Vol. 17. Elsevier Science Ltd., Amsterdam, New York, USA., ISBN: 13: 978-0444989109, pp: 176.

Mathon, C. and A. Limam, 2006. Experimental collapse of thin cylindrical shells submitted to internal pressure and pure bending. Thin-Walled Struct., 44: 39-50. DOI: 10.1016/j.tws.2005.09.006

Pircher, M., P.A. Berry, X. Ding and R.Q. Bridge, 2001. The shape of circumferential weld-induced imperfections in thin-walled steel silos and tanks. Thin-Walled Struct., 39: 999-1014. DOI: 10.1016/S0263-8231(01)00047-7

Taguchi, G., 1986. Introduction to Quality Engineering: Designing Quality into Products and Processes. Illustrated Edn., Quality Resources, ISBN: 13: 9789283310839, pp: 191.

Yamaki, N., 1984. Elastic Stability of Circular Cylindrical Shells. Elsevier Science Pub. Co., Amsterdam, New York, USA., ISBN: 10: 0444868577, pp: 558. 